\title{
Performance Analysis of different Digital Modulation/Demodulation Based on BERTool
}

\author{
Jihua Huang ${ }^{1}$, Shikai Shen ${ }^{2}$, Jin Wen ${ }^{1}$,Xingyuan Chen ${ }^{1}$ \\ 1. School of Information Technology, Kunming University, 650214, China \\ 2. Center for modern educational techonolgy, Kunming University, 650214, China \\ Jihua_huang@yeah.net
}

\begin{abstract}
Keywords: Modulation; BPSK; QPSK; OQPSK; QAM; MSK; Bit Error Rate (BER)
\end{abstract}
\begin{abstract}
Modulation enable us to transmit information at different frequencies. It plays a crucial role in transmit signals in communication systems. In this work, the performance of various modulation schemes under AWGN channel, Rayleigh fading channel and Rician fading channels are investigated. The performance comparison among PSK, OQPSK, PAM, QAM, DPSK, and MSK modulation is studied base on BERTool and the visual results shows that under the same transmission bandwidth and the same bit error probability, the QAM and MSK modulation has a better performance compared with other modulation method.
\end{abstract}

\section{Introduction}

In digital communication systems, the source to be transmitted is discrete both in time and amplitude. Digital information carrying signals must be firstly converted to an analog waveform prior to transmission, and at the receiving end, analog signals are converted back to a digital format before presentation to the end user. The conversion process at the transmitting end is known as modulation, and the receiving end is known as demodulation or detection [1].

Because modulation plays an important role in transmission of signals from transmitter to receiver in all communication systems, especially for wireless communication, many efforts has focused on these areas [7][8][9]. In digital transmission, the performance of digital modulation systems is of the most important key factors to achieve the best results of the communication process [2]. So here, we concentrate on analysis of the bit error rate (BER) of digital communication system.

In this paper presents a simulation study of some important digital modulations, such as, Binary Phase Shift Keying (BPSK), Quadrature Phase Shift Keying (QPSK) and Offset Quadrature Phase Shift Keying (OQPSK), based on Matlab BERTool and with the aid of a Simulink model. Comparison of the Mont Carlo performance using MATLAB program.

\section{Ensemble learning based prediction model}

\subsection{Binary Phase Shift Keying (BPSK) Data processing}

One of the simplest forms of digital modulation is binary or Bi-Phase Shift Keying (BPSK). BPSK is a two phase modulation scheme, where the 0's and 1's in a binary message are represented by two different phase states in the carrier signal:

$$
s(t)= \begin{cases}A \cos \left(2 \pi f_{c} t\right) & \text { binary } 1 \\ -A \cos \left(2 \pi f_{c} t\right) & \text { binary } 0\end{cases}
$$

In equation (1), $A$ is amplitude, and $f_{c}$ is carry frequency. One application where this is used is for deep space telemetry.

For coherent detection, non-selective Linear Time-Invariant (LTI) channels, the BER of BPSK under additive white Gaussian noise (AWGN) is:[3] 


$$
P_{e}=\frac{1}{2} \operatorname{erfc}\left(\sqrt{\frac{E_{b}}{N_{o}}}\right)
$$

where $E_{b}$ is energy per bit, $N_{o}$ is the on-sided spectral power spectral density, $E_{b} / N_{o}$ is the signalto-noise ratio, and $\operatorname{erfc}$ is complementary error function, $\operatorname{erf} c(k)=2 Q(\sqrt{2} k)$.

Another PSK modulation is Differential Phase Shift Keying (DPSK). In DPSK, the phase of the modulated signal is shifted relative to the previous signal element [3]. This modulation can overcome the problem when differential encoding and phase shift keying are combined together at the transmitter. In this, the phase is unchanged for the transmission of the symbol 1 , whereas the phase of the signal is advanced by for the transmission of symbol 0 .

\subsection{Quadrature Phase Shift Keying (QPSK)}

QPSK uses two basic functions, a sine and cosine whereas BPSK uses just on. By varying the phase of each carries $(0,90,180$, or 270 degrees), the communication system can send two bits per each signal. QPSK is a two-dimensional signal, due to the fact that it sends two independent signals to create the symbols. It allows the signal to carry twice as much information as an ordinary PSK. The application of QPSK is multiple. For example, it can be used for satellite transmission of MPEG2 video, cable modems, Videoconferencing, cellular phone systems, and other forms of digital communication over an RF carrier [2].

\subsection{Offset Quadrature Phase Shift Keying (OQPSK)}

OQPSK modulation technique is the spectral characteristics of the constant envelope modulation techniques, and has a very high spectral efficiency and power utilization [5]. This modulation technique is a variant of phase shift keying using four different values of the phase to transmit. Because the insertion of a delay in the even data stream is the only difference between a conventional QPSK modulator and OQPSK, the OQPSK has the same error performance as QPSK[5]:

$$
P_{e}=\frac{1}{2} \operatorname{erfc}\left(\sqrt{\frac{E_{b}}{N_{o}}}\right)
$$

\subsection{Quadrature Amplitude Modulation (QAM)}

The QAM is a modulation scheme where its amplitude is allowed to vary with phase [6]. This technique can be viewed as a combination of ASK as well as PSK [7].

In QAM modulation scheme, the probability of error is given as [8]:

$$
P_{e}=\frac{3}{4} Q\left(\sqrt{\frac{4 E_{b}}{2(M-1) N_{0}}}\right)^{2}-\frac{9}{16} Q^{2}\left(\sqrt{\frac{2 E_{b}}{7 N_{0}}}\right)
$$

where the $Q$ function is defined as: $Q(x)=\frac{1}{\sqrt{2 \pi}} \int_{x}^{\infty} e^{-\frac{x^{2}}{2}} d x$.

\subsection{Minimum Shift Keying (MSK)}

Minimum Shift Keying (MSK) is one of the most spectrally efficient modulation schemes available. It is equivalent to OQPSK and encoded with bits alternating between quadrature components, 
with the Q component delayed by half the symbol period. It encodes each bit as a half sinusoid.

If we define the spreading waveform in a subchip of length $T_{s c}$ where $T_{c}=n T_{s c}$, being $T_{c}$ the duration of a chip and $\mathrm{n}$ the number of subchips in one chip. Accordingly, this is the Fourier transform of the subchip part and $f_{s c}=n f_{c}$ then the normalized Power Spectral Density of the spreading MSK waveform can be expressed as follows [8]:

$$
G_{M S K}(f)=\frac{8 f_{s c}^{3}}{\pi^{2}} \frac{\cos ^{2}\left(\pi f / f_{s c}\right)}{\left(f_{s c}^{2}-4 f^{2}\right)^{2}}
$$

\section{BER over fading channel}

A fading channel can be considered as an AWGN with a variable gain. The gain itself is considered as a RV with a given pdf. So the average BER can be calculated by averaging BER for instantaneous SNR over the distribution of SNR. The BER is expressed by a Q-function as following [9]:

$$
P_{e}=\int_{0}^{\infty} Q(\sqrt{2 g \gamma}) p_{\gamma}(\gamma) d \gamma
$$

where $g=1$ for the case of coherent BPSK.

\section{Simulation and Results}

BERTool is an interactive GUI for analyzing bit error rate (BER) performance of communication system [9]. Using we can do many things, such as: Generate BER data for a communication system; Closed - form expressions for theoretical BER performance of selected types of communication system; Calculating BER using semi- analytical technique [10].

\subsection{Theoretical Data Simulation}

In this section, we use BERTool to generate and analyze theoretical BER data. In Figure 1, we compare theoretical BER of PSK and DPK modulation. The simulation is under AWGN channel. From the Figure 1, we can see, DPSK modulation has higher BER. In Figure 2, we compare theoretical BER of DPSK and OQPSK modulation under Rayleigh fading channel.

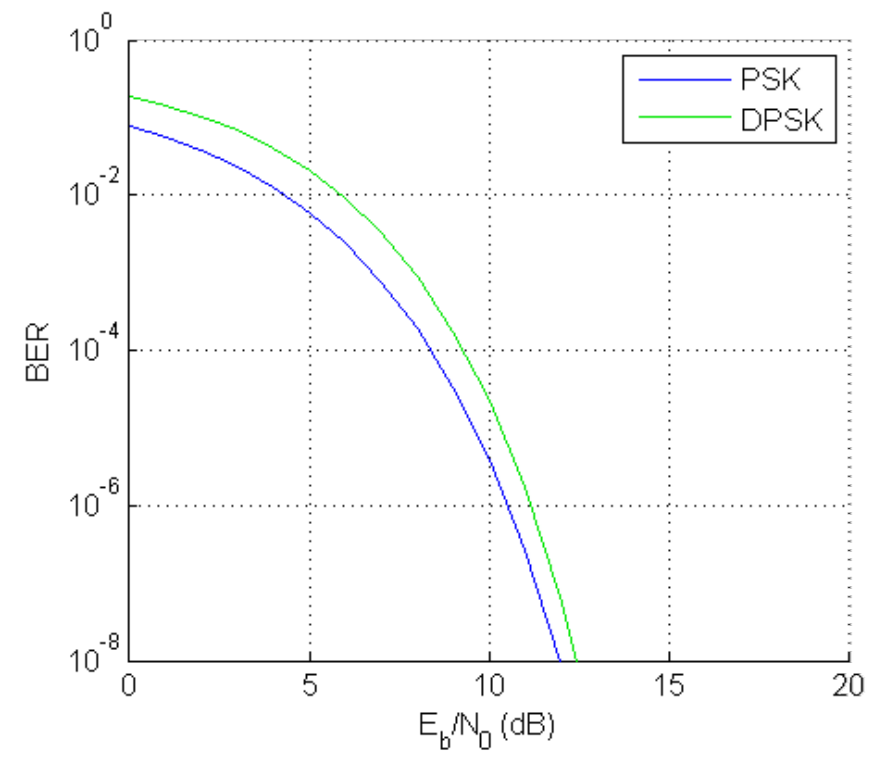

Figure 1. BER Comparison of PSK \& DPSK under AWGN Channel 


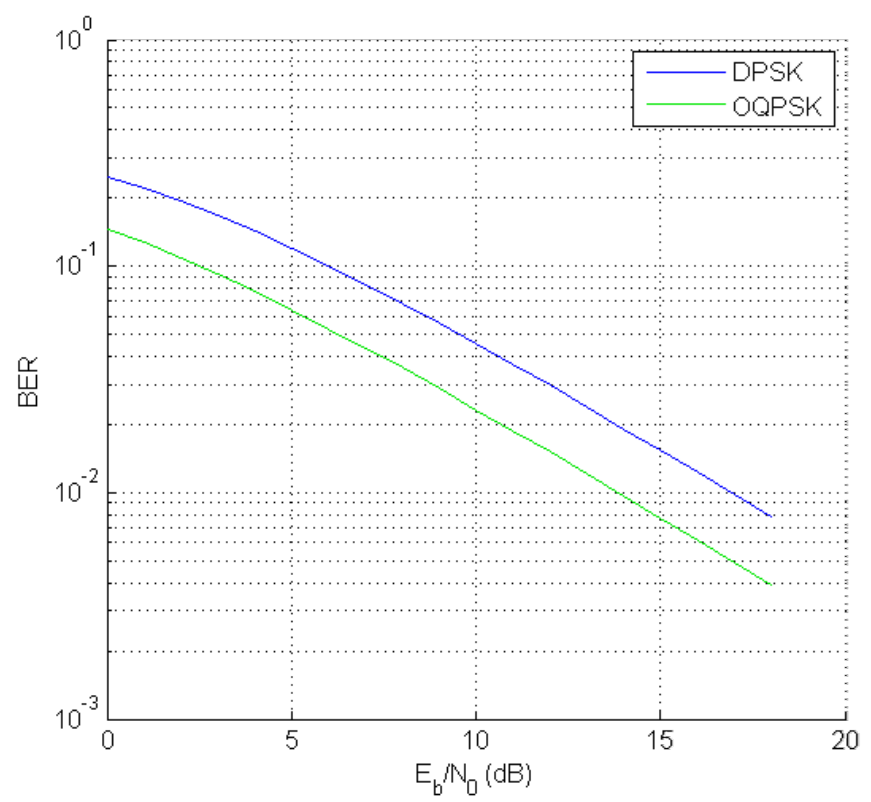

Figure 2. BER Comparison of DPSK \& OQPSK under Rayleigh Channel

From the Figure 1, we can see, DPSK modulation has higher BER. And from the Figure 2, we can see DPSK modulation has higher BER under Rayleigh fading channel.

\subsection{Semi Analytic Simulation}

In this section, we give the simulation results of different orders of QAM based on semi analytic technique.

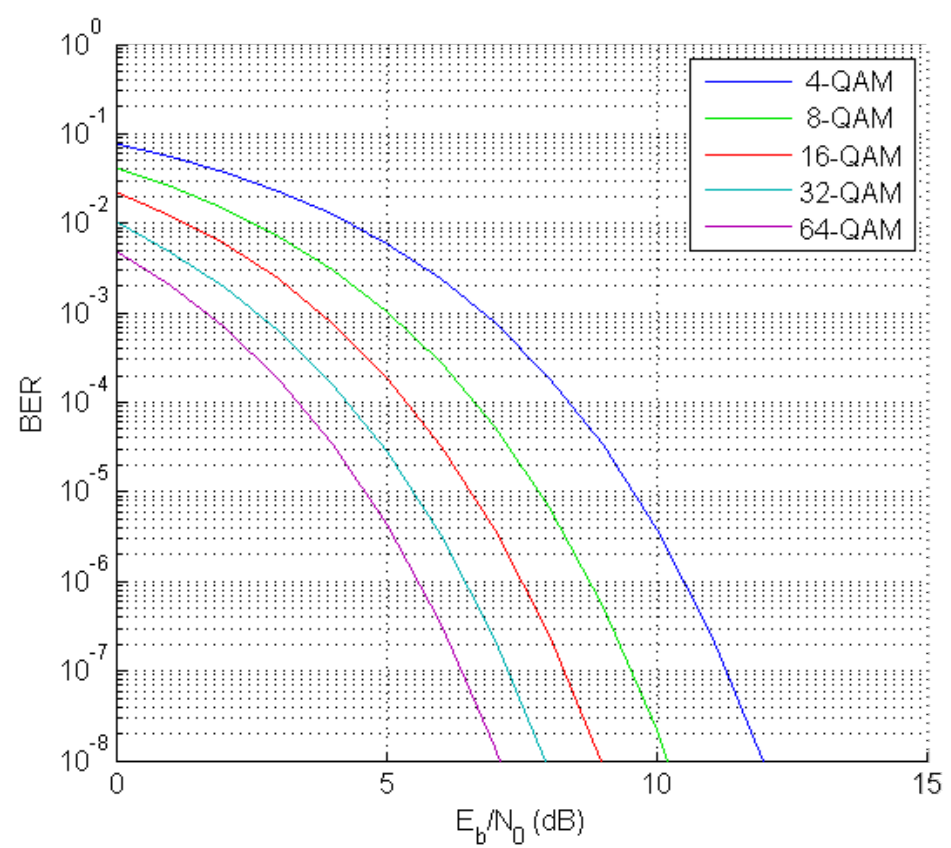

Figure 3. BER Comparison between different between orders of QAM Modulation 


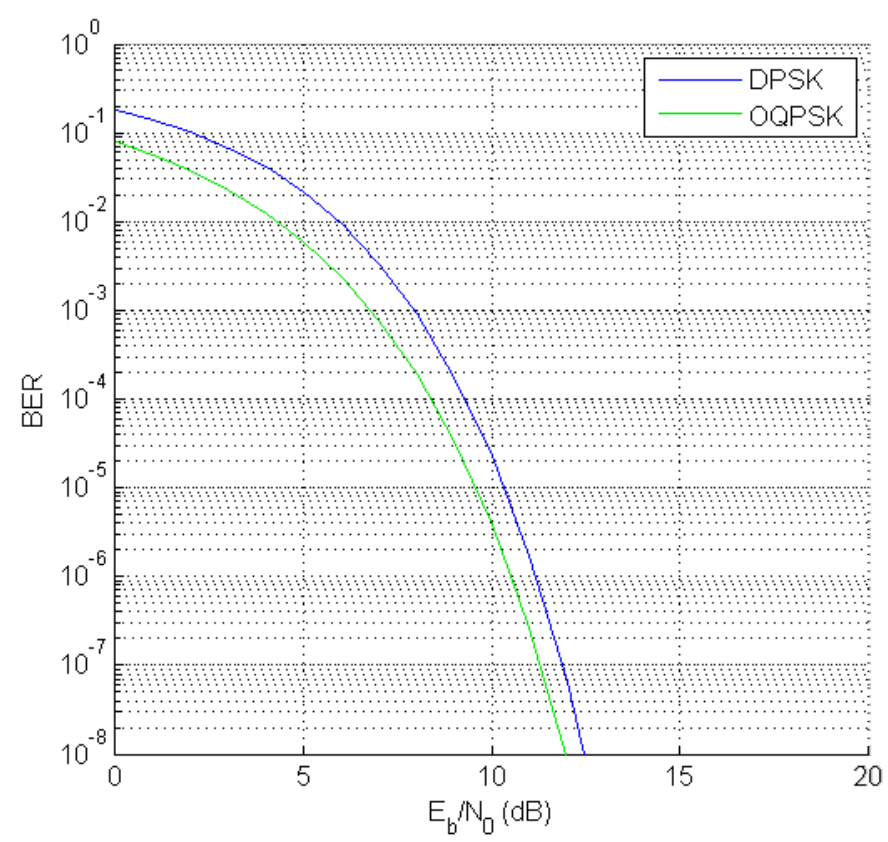

Figure 4. BER Comparison DPSK \& OPSK Modulation

From Figure 3 we can see that with the increasing of QAM order, the BER value is dropping. From Figure 4 we can see that OQPSK modulation has lower performance compared with DPSK modulation when using the semi analytic technique under AWGN Channel.

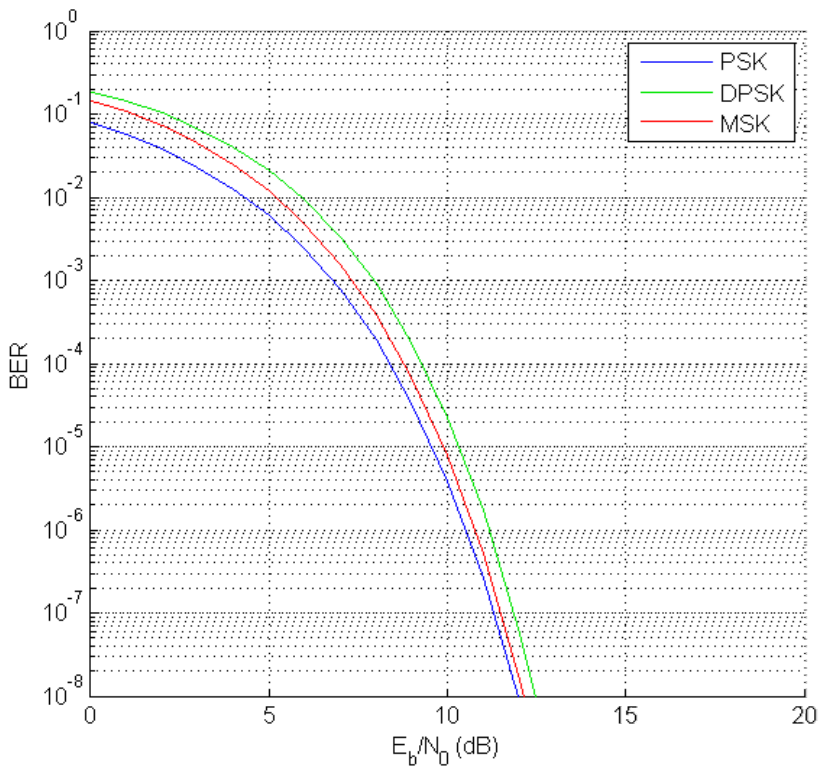

Figure 5. BER Comparison between PSK, DPSK \& MSK Modulation

From Figure 5 we can see that MSK modulation has the best performance when using semi analytic technique. In this simulation, the signal is under AWGN channel, and the order of all three modulations is 2 .

\subsection{Monte Carlo Simulation}

In this section, we use BERTool in conjunction with simulation model to generate and analyze BER data. In Figure 6, we compare the 16 - QAM modulation BER value with the simulation value based on Monte Carlo method. From Figure 5 we can see that the semi analytic BER value of 16QAM modulation fits well with the value simulated using the Monte Carlo method. 
Monte Carlo simulation is a broad class of computational algorithms that rely on repeated random sampling to obtain numerical results. It performs risk analysis by building models of possible results by substituting a range of values for any factor that has inherent uncertainty. It then calculates results over and over, each time using a different set of random values from the probability functions [11].

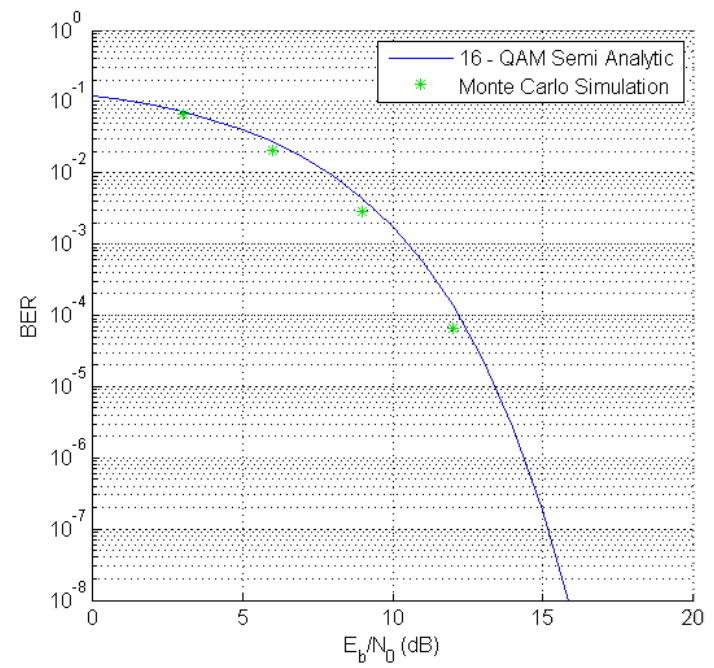

Figure 6. BER Comparison between 16-QAM Semi Analytic \& Monte Carlo Simulation

\subsection{Simulink BER Calculation}

In this section, we compare the BER performance of a Simulink simulation with theoretical BER results. We use GMSK in this simulation and the modulated signal goes through Rician fading channel. The Figure 7 is the GMSK modulation Simulink model, in the model, buffers are used to realize serial-to-parallel transformation. Simulation results is stored in the workspace and be used to do theoretical simulation in BERTool. Visual results curves are shown in Figure 8. From Figure 8 we can see that the GMSK theoretical simulation result fits well with the Monte Carlo simulation.

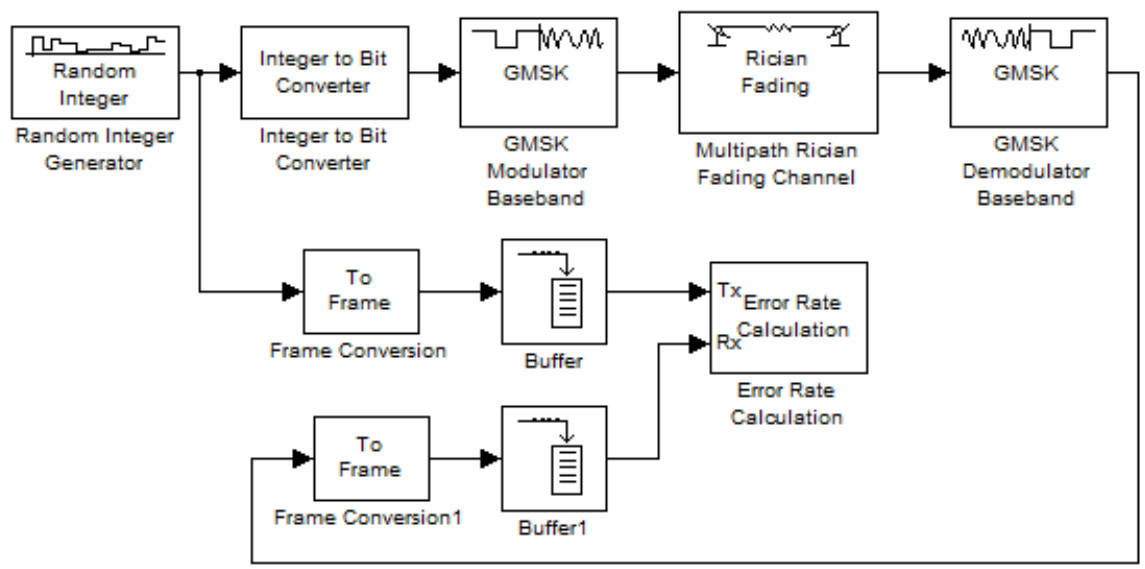

Figure 7. GMK Simulink Simulation under Rician Fading Channel 


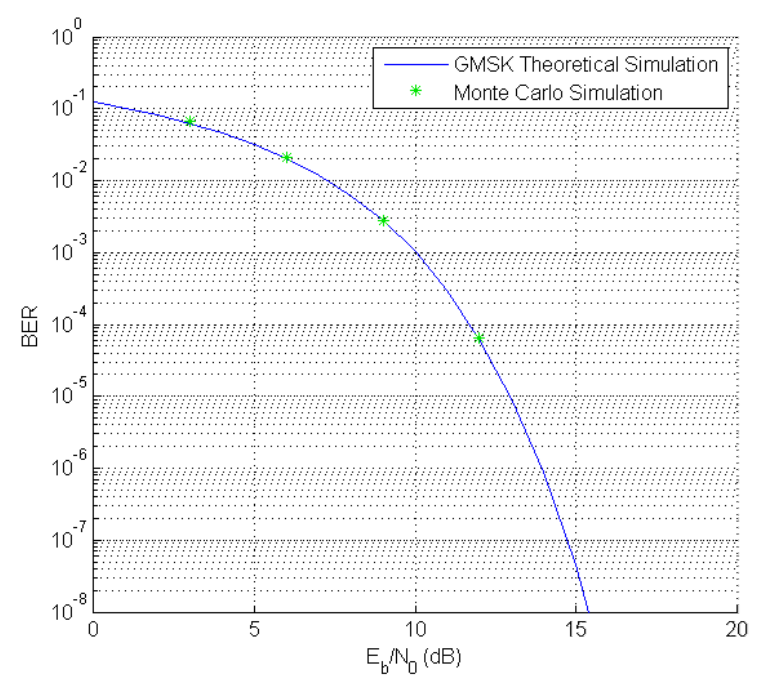

Figure 8. BER Comparison between GMSK Theoretical Simulation \& Monte Carlo Simulation

\section{Conclusion}

Modulation technology is important in all kinds of communication systems, especially for today's wireless communication systems. Because it contributes to the evolution of mobile communications by increasing the capacity, speed as well as the quality of the wireless network. Without an appropriate modulation scheme, it would be impossible to achieve an expected throughput, and it is impossible to realize a reliable, robust and efficient wireless communication system. BER is a key parameter that is used in assessing systems that transmit digital data from one location to another.

A simulation study based on BERTool on the digital modulation techniques under AWGN, Rayleigh fading channel and Rician fading channel presented in this paper reveals that the selection of digital modulation technique has better performance over other modulation methods on the type of specific application. Standard Monte Carlo simulation techniques are also presented when analyze GMSK modulation system, the result shows that the theoretical simulation fits perfectly with the Monte Carlo simulation result.

\section{Acknowledgments}

This work was supported by the Scientific and Technological Innovation Team of IOT Application Technology of Kunming(No. 2016-2-R-07793), Scientific Research and Innovation Team of Kunming University based on IOT Technology(No. 2015CXTD04), Reform and innovation Project of Application-oriented Talents Training of Kunming University in 2015-Construction of practical training base in computer major(2015-2018), and the School Science Foundation (XJZD1609): "The Applied Research of ZigBee Technology in Intellectual Residential System”.

\section{References}

[1]https://en.wikipedia.org/wiki/Digital_Modulation.

[2]Amer Mohamed Daeri, Mohamed Elfituri, and Amer Ragab Zerek, Quadrature Phase Shift Keying and Offset Quadrature Phase Shift Keying BER Performance Comparison. Proceedings of Engineering \& Technology. Pp.934-939.

[3]https://en.wikipedia.org/wiki/Bit_error_rate.

[4]Communications Systems, H. Stern \& S. Mahmoud, Pearson Prentice Hall, 2004, p. 283.

[5] HuXiao, The Application of OQPSK Synchronizatipn Algorithm in IEEE802.15.4.Master's thesis, 2013.

[6]S.Haykin, M. Moher, Introduction to Analog and Digital Communications, John Wiley \& Sons, 
Inc., 2007.

[7]M.A.Masud, M.Samsuzzaman, M.A.Rahman, Bit Error Rate Performance Analysis on Modulation Techniques of Wideband Code Division Multiple Access, Journal of Telecommunications, Vol.1, Issue 2, March 2010.

[8]Jonqyin (Russell) Sun "Linear diversity analysis for QAM in Rician fading channels", IEEE WOCC 2014.

[9]http://www.palisade.com/risk/monte_carlo_simulation.asp

[10] https://ww2.mathworks.cn/help/comm/ref/bertool.html.

[11] P.Aulin,W.C.Sundberg, Continuous Phase Modulation Part-1: Full Response Signaling, IEEE Transactions on Communications, Vol. COM-29, No. 3, 1981, pp 196-209. 